\title{
Blurred lines for management of thyroid nodules in the era of atypia of undetermined significance/ follicular lesion of undetermined significance: novel subdivisions of categories IIIA and IIIB in a possible forthcoming The Bethesda System for Reporting Thyroid Cytopathology, 3rd edition; amending versus unnecessary?
}

\author{
Ilker Sengul1,2 (1), Demet Sengul3* (1)
}

The management of indeterminate cytology ${ }^{1-5}$ of the thyroid nodules, particularly the atypia of undetermined significance or follicular lesion of undetermined significance (AUS/FLUS), has still been one of the most challenging issues in endocrine pathology, neck endocrine surgery, endocrine surgery, endocrinology, and thyroidology. Of note, the estimated risk of malignancy (ROM) for AUS/FLUS was 5-15\% in the 1st edition (ed.) of The Bethesda System for Reporting Thyroid Cytopathology (TBSRTC), whereas it was increased to $10-30 \%$ in the 2 nd ed. TBSRTC. The 2015 American Thyroid Association (ATA) management guidelines for adult patients with thyroid nodules and differentiated thyroid cancer recommend repeat fine-needle aspiration (FNA), molecular testing, surveillance, or diagnostic lobectomy for nodules with AUS/FLUS cytology, after consideration of worrisome clinical and sonographic features ${ }^{3,4}$. The 2015 ATA management guidelines also recommend informed patient preference and feasibility in clinical decision-making ([A17] AUS/FLUS cytology, Recommendation 15A, Weak recommendation-Moderate quality evidence) $)^{3}$. Deftereos and colleagues ${ }^{4}$ recently reported a worthy study, entitled "Differential outcomes of patients with thyroid FNA diagnoses of AUS/FLUS with and without nuclear atypia: The potential need for separation in the Bethesda System.” They proclaimed that the presence or absence of the nuclear atypia is pertinent to the different malignancy rates and, therefore, propounded Category III, TBSRTC might be being divided into two subcategories with different implied ROMs by virtue of the presence of nuclear atypia (consisting of intranuclear pseudoinclusions, nuclear grooving, irregular nuclear contours, and nuclear overlapping). The 2014 Italian Consensus for the Classification and Reporting of Thyroid Cytology (ICCRTC) divided diagnostic category TIR3, indeterminate cytology, into two subcategories, namely, TIR3A (low-risk indeterminate lesion) and TIR3B (high-risk indeterminate lesion), with different ROMs and diverse clinical behaviors. TIR3A is characterized by augmented cellularity with numerous microfollicular structures in a background of poor colloid or scarce cellular structure including predominantly microfollicular groups, also with oxyphilic features, Hurthle cells, harboring estimated ROM of $<10 \%$, whereas TIR3B is proclaimed cases with "mild/focal nuclear atypia" at expected higher ROM of 15-30\%. Even though

(i) Thy 3A, neoplasm possible, atypia/nondiagnostic, the Royal College of Pathologists, United Kingdom (RCPath, UK);

(ii) TIR3A, low-risk indeterminate lesion, ICCRTC;

(iii)indeterminate or follicular lesion of undetermined significance, Thyroid Cytology Structured Reporting Protocol, 2014 Royal College of Pathologists of Australia (RCPA, 2014); and

\footnotetext{
${ }^{1}$ Giresun University, Faculty of Medicine, Division of Endocrine Surgery - Giresun, Turkey.

${ }^{2}$ Giresun University, Faculty of Medicine, Department of General Surgery - Giresun, Turkey.

${ }^{3}$ Giresun University, Faculty of Medicine, Department of Pathology - Giresun, Turkey.

*Corresponding author: demet.sengul.52@gmail.com

Conflicts of interest: the authors declare there are no conflicts of interest. Funding: none.

Received on August 10, 2021. Accepted on August 14, 2021.
} 
(iv) AUS/FLUS, TBSRTC, 2nd ed. have been compared and matched as if being in the equivalent status, the ROMs are not completely the same for determining the mentioned groups of indeterminate cytology in terms of AUS/FLUS.

For instance, they are $<10 \%$ for TIR3A, ICCRTC and $10-30 \%$ for AUS/FLUS, the 2 nd ed. TBSRTC 5 . Of note, actually, the ROM value of TIR3B (15-30\%) is compatible with AUS/FLUS (10-30\%) rather than TIR3A. As already known, TIR3B involves some additional features consistent with papillary thyroid carcinoma, such as nuclear inclusion, nuclear groove, and overlapping nucleus. To this end, the suggested actions also differ for both TIR3A and TIR3B, i.e., repeat FNA/clinical follow-up for indeterminate cytology whereas surgery for indeterminate cytology with nuclear alterations. Finally, the recommended usual management for AUS/FLUS is repeat FNA, molecular testing, or lobectomy in the 2 nd ed. TBSRTC, compatible with both TIR3A and TIR3B, in spite of propounding ROM of Category III, TBSRTC, 2nd ed., is consonant with TIR3B, ICCRTC ${ }^{5,6}$. We recently emphasized whether it is essential to maintain Category III, TBSRTC as a unique and indivisible category, per se, among indeterminate cytology of thyroid nodules or not, published in Volume 67, Revista da Associação Médica Brasileira ${ }^{7}$. We currently have mentioned Category III from another perspective and recommended the requirement of zooming in thyroid nodules in suspense, $10-15 \mathrm{~mm}$ with repeat cytology, Category III, TBSRTC in Volume 67, Revista da Associação Médica Brasileira7.
In conclusion, it is critical for the endocrine surgeons and thyroidologists, who stay informed of the growing spectrum of clinical presentation for Category III of TBSRTC, AUS/ FLUS cytology, to ensure appropriate clinical care and use of FNA cytology in order to minimize overlooking thyroid malignancy. We postulated that the so-called subdivision concept in Category III, TBSRTC:

(i) Category IIIA: AUS/FLUS without nuclear atypia (AUS/ FLUS wo NA) and

(ii) Category IIIB: AUS/FLUS with nuclear atypia (AUS/ FLUS w NA)

Within the possible forthcoming Category III, TBSRTC, 3rd ed., the 202X TBSRTC, with different newly established ROMs for each diagnostic category might selectively enrich the different management proposals in thyroidology. As a matter of fact, this issue merits further investigation.

\section{ACKNOWLEDGMENT}

The authors thank all of the study participants.

\section{AUTHORS" CONTRIBUTIONS}

IS: Conceptualization, Data curation, Formal Analysis, Writing original draft, Writing - review \& editing. DS: Conceptualization, Data curation, Formal Analysis, Writing - original draft, Writing - review \& editing.

\section{REFERENCES}

1. Sengul D, Sengul I. Association between Tsukuba elasticity scores 4 and 5 on elastography and Bethesda undetermined cytology on US-guided FNA with 27-G needle, verified by histopathology: a cut-off point of $20 \mathrm{~mm}$ of diameter designated for thyroid nodules. J BUON. 2019:24(1):382-90. PMID: 30941995

2. Sengul D, Sengul I, Van Slycke S. Risk stratification of the thyroid nodule with Bethesda indeterminate cytology, category III, IV, V on the one surgeon-performed US-guided fine-needle aspiration with 27-gauge needle, verified by histopathology of thyroidectomy: the additional value of one surgeon-performed elastography. Acta Chir Belg. 2019;119(1):38-46. https://doi. org/10.1080/00015458.2018.1551769

3. Sengul D, Sengul I, Pelikán A. Paraphrase for the impact of repeat fine-needle aspiration in thyroid nodules categorized as atypia of undetermined significance or follicular lesion of undetermined significance: a single center experience. Diagn Cytopathol. 2021;49(3):452-3. https://doi.org/10.1002/ dc. 24685
4. Deftereos G, Schmechel SC, Waner EE, Itani M, Dighe MK, Tylee TS. Differential outcomes of patients with thyroid FNA diagnoses of AUS/FLUS with and without nuclear atypia: the potential need for separation in the Bethesda System. Diagn Cytopathol. 2020;48(7):610-7. https://doi.org/10.1002/ dc. 24424

5. Nardi F, Basolo F, Crescenzi A, Fadda G, Frasoldati A, Orlandi $\mathrm{F}$, et al. Italian consensus for the classifcation and reporting of thyroid cytology. J Endocrinol Invest. 2014;37(6):593-9. https://doi.org/10.1007/s40618-014-0062-0

6. Desai $D$, Lepe $M$, Baloch ZW, Mandel SJ. ThyroSeq v3 for Bethesda III and IV: an institutional experience. Cancer Cytopathol. 2021;129(2):164-70. https://doi.org/10.1002/ cncy. 22362

7. Sengul I, Sengul D. Focusing on thyroid nodules in suspense: 10-15 mm with repeat cytology, Category III, the Bethesda System for Reporting Thyroid Cytopathology, TBSRTC. Rev Assoc Med Bras (1992). 2021;67(2):166-7. https://doi. org/10.1590/1806-9282.67.02.20200828 